\title{
Criminologie
}

\section{Les droits des mineurs et l'emprisonnement des jeunes au Québec}

\section{Alice Parizeau}

Volume 9, numéro 1-2, 1976

L’emprisonnement au Québec

URI : https://id.erudit.org/iderudit/017054ar

DOI : https://doi.org/10.7202/017054ar

Aller au sommaire du numéro

Éditeur(s)

Les Presses de l'Université de Montréal

ISSN

0316-0041 (imprimé)

1492-1367 (numérique)

Découvrir la revue

Citer cet article

Parizeau, A. (1976). Les droits des mineurs et l'emprisonnement des jeunes au Québec. Criminologie, 9(1-2), 118-147. https://doi.org/10.7202/017054ar d'utilisation que vous pouvez consulter en ligne.

https://apropos.erudit.org/fr/usagers/politique-dutilisation/ 
LES DROITS DES MINEURS ET L'EMPRISONNEMENT DES JEUNES AU QUÉBEC

Ả une certaine époque sociologues et économistes, ont prétendu que le potentiel collectif du développement dépendait du degré de l'industrialisation. Désormais, des chefs d'écoles, tels que François Perroux ou Alfred Sauvy, reconnaissent que c'est l'homme qui détermine le véritable potentiel de toute société.

\section{A. LA SOCIETÉ ET L'ENFANCE}

Certes dans les pays du Tiers-Monde, le fait que 89 millions d'enfants naissent chaque année, est un facteur important des pénuries, il n'en reste pas moins que même là, on doit tenir compte de l'évolution culturelle que favorise la pénétration dans la société des cohortes des jeunes mieux formés et mieux préparés à affronter les impératifs de l'industrialisation. Si les mineurs âgés de moins de 15 ans, qui représentent dans le Tiers-Monde une population d'un milliard 52 millions ne parviennent pas à remplir la mission qui leur revient de fait, c'est là une conséquence et non pas la cause du sous-développement.

Autant, toutefois, il est justifiable que des collectivités qui affrontent la deuxième partie du XX' sieccle avec un sérieux retard ne parviennent pas à assumer certaines responsabilités relatives à la formation des jeunes générations, autant tel a'est pas le cas pour les pays industrialisés.

En premier lieu, dans ces pays la croissance est beaucoup plus faible et le taux annuel de naissances ne dépasse pas 19 millions, tandis que le total des jeunes de moins de 15 ans ne représente que quelque 93 millions. 
TABLEAU 1

Statistiques de la population

(Annuaire du Canada)

Superficie du territoire et densité de la population par province, années de recensement 1951-71

\begin{tabular}{|c|c|c|c|c|c|c|}
\hline \multirow[t]{2}{*}{ Province au territoire } & \multirow{2}{*}{$\begin{array}{l}\text { Superficie } \\
\text { milles carrés }\end{array}$} & \multicolumn{5}{|c|}{ Habitants par mille carré } \\
\hline & & 1951 & 1956 & 1961 & 1966 & 1971 \\
\hline Terre-Neuve & 143045 & 2.53 & 2.90 & 3.20 & 3.45 & 3.65 \\
\hline lle-du-Prince-Édouard & 2184 & 45.07 & 45.46 & $47.9 \mathrm{~J}$ & 49.70 & 51.11 \\
\hline Nouvelle-Écosse & 20402 & 31.50 & 34.05 & 36.12 & 37.06 & 38.67 \\
\hline Nouveau-Brunswick & 27835 & 18.53 & 39.93 & 21.48 & 22.16 & 22.80 \\
\hline Québec & 523860 & 7.74 & 8.84 & 10.04 & 11.04 & 11.50 \\
\hline Ontario & 344092 & 13.36 & 15.71 & 18.12 & 20.23 & 22.39 \\
\hline Manitoba & 211775 & 3.67 & 4.01 & 4.35 & 4.55 & 4.66 \\
\hline Saskatchewan & 220182 & 3.78 & 4.00 & 4.20 & 4.34 & 4.21 \\
\hline Alberta & 248800 & 3.78 & $4.5 i$ & 5.35 & 5.88 & 6.54 \\
\hline Colombie-Britannique & 369279 & 3.24 & 3.89 & 4.53 & 5.22 & 6.08 \\
\hline Canada (sans les Territoires) & 2101454 & 6.65 & 7.64 & 8.66 & 9.50 & 10.24 \\
\hline Yukon & 205346 & 0.04 & 0.06 & 0.07 & 0.07 & 0.09 \\
\hline Territoires du Nord-Ouest & 1253438 & 0.01 & 0.02 & 0.02 & 0.02 & 0.03 \\
\hline Canada & 3560238 & 3.93 & 4.52 & 5.12 & 5.62 & 6.06 \\
\hline
\end{tabular}


Au Canada, plus spécifiquement, où le taux de croissance naturelle est relativement faible et où d'une façon générale la densité de la population par kilomètre carré est exceptionnellement basse, les mineurs présentent pour la population active, entre autre, un fardeau sensiblement allégé comparativement à celui que doivent assumer par exemple, les collectivités européennes.

Sommes-nous conscients collectivement de l'importance de cette "richesse naturelle» fondamentale que constitue pour le Canada, tout citoyen mineur?

Malgré certaines déclarations des hommes publics, dont Lester B. Pearson, par exemple, Prix Nobel de la paix, il semble bien que les politiques des gouvernements canadiens, fédéral et provinciaux, n'ont pas été sensiblement influencées, par cet impératif.

En dehors d'un certain nombre de vœux pieux, on chercherait en vain, en effet, l'amorce d'une philosophie de défense des intérêts des mineurs en tant que tels. Conformément à la tendance qui prévaut dans l'ensemble des pays occidentaux, on parle surtout de l'aide à la famille, mais rarement, sinon jamais, des droits des mineurs. C'est à se demander même si les mineurs, qui constituent la masse des citoyens qui ne votent pas, ont vraiment des droits dans notre société, ou uniquement des devoirs.

En effet, en ce qui a trait aux devoirs des mineurs, ils sont assez clairement précisés dans les codes.

C'est ainsi que selon le Code Civil, "L'enfant à tout âge doit honneur et respect à ses père et mère" (art. 242. Titre huitième. De la puissance paternelle). On précise, par la suite, à l'article 244,245 et $245 \mathrm{a}$, ce qui suit :

Le mineur non émancipé ne peut quitter la maison paternelle sans la permission de son père" (art. 244). "Le père, et à son défaut la mère, a sur son enfant mineur et non émancipé un droit de correction modéré et raisonnable, droit qui peut être délégué et que peuvent exercer ceux à qui l'éducation de cet enfant a été confiée" (art. 245). "Les parents exercent à l'égard de leur enfant naturel mineur et non émancipé qu'ils n'ont pas abandonné, les pouvoirs et l'autorité dont ils ont besoin pour s'acquitter de leurs obligations envers lui... (art. 245a).

Par ailleurs, les enfants ont l'obligation de «fournir à leurs parents indigents, les nécessités de la vie» (chap. IV, art. 1057). 
En ce qui a trait aux parents, par contre, ils sont chargés de dédommager le cas échéant les tiers puisque «Le père et après son décès, la mère, sont responsables du dommage causé par leurs enfants mineurs...D (art. 1054, chap. III).

En somme, à l'intérieur de la famille, entre les parents et les enfants, le législateur a confirmé le lien naturel de dépendance tout en imposant à la famille des responsabilités à l'égard de la collectivité. Dès lors, le mineur n'a pas de droits face à cette collectivité, mais uniquement le devoir de respecter ses règles; devoirs qu'on peut qualifier, à certains égards, d'exorbitants.

En effet, selon la loi, est considéré comme jeune délinquant tout enfant «qui commet une infraction à quelqu'une des dispositions du Code criminel, ou d'un statut fédéral ou provincial, ou d'un règlement ou ordonnance d'une municipalité, ou qui est coupable d'immoralité sexuelle ou de toute forme semblable de vice, ou qui, en raison de toute autre infraction, est passible de détention dans une école industrielle ou maison de correction pour les jeunes délinquants en vertu d'un statut fédéral ou provincial» (Loi sur les jeunes délinquants, chap. J-3), art. 2).

Comme on le constate, notre collectivité se protège contre les agissements des mineurs contraires à l'ordre, ou aux normes établies, de façon qui permet d'inclure toutes les formes de comportements qui ne correspondent pas à ses règles.

À travers la Loi de protection de l'enfance et de la jeunesse, qui est du ressort provincial, la collectivité admet uniquement que les mineurs qui ne reçoivent pas dans leur famille les soins voulus, ou qui n'ont pas de famille, peuvent être placés sous la protection de la Cour du bien-être social. Il s'agit là, toutefois, d'une protection limitée à certaines catégories spécifiques des mineurs et qui n'autorise l'intervention de la société que dans des situations plus ou moins critiques, où on constate l'indifférence, la cruauté ou l'incapacité de la famille à remplir sa fonction fondamentale de défenseur naturel de l'enfant.

Étant donné, en outre, que dans la législation canadienne on ne prévoit pas de loi régissant la déchéance des droits de paternité, et que les parents criminels peuvent continuer à garder leurs droits sur un enfant, même quand il est placé sous la protection de la Cour, l'enfant demeure en fait, à leur merci.

Certes, la Cour peut condamner des parents criminels à deux ans de prison, ou à cinq cents dollars d'amende, mais cela ne règle 
pas pour autant les problèmes relatifs à l'émancipation du mineur, ou à la possibilité de donner un enfant à l'adoption.

En somme, dans toutes les situations comme en toute circonstance, notre société se préoccupe principalement des droits des parents et même quand elle est obligée, à la suite des circonstances particulièrement pénibles, d'intervenir pour protéger le mineur, on continue à tenir compte des prérogatives parentales. Cet état de fait n'est pas d'ailleurs le propre de notre collectivité, mais prévaut dans la plupart des pays. Les législations diffèrent, mais la philosophie demeure la même.

\section{B. LES DROITS DES MINEURS ET LE CONCEPT DE PROTECTION COLLECTIVE}

En ce qui a trait aux droits des mineurs, par contre, ils s'accompagnent toujours d'une certaine forme de contrainte. C'est ainsi que les enfants ont droit à une éducation scolaire gratuite, mais en même temps obligatoire. Cela signifie qu'ils doivent "profiter" de ce droit sous peine de sanctions. Les autres droits des enfants, contrairement aux droits de l'homme, largement discutés et largement divulgués dans les pays occidentaux, demeurent un ensemble de bonnes intentions.

Formulée officiellement en 1959 dans le rapport de la Troisième Commission des Nations unies, la déclaration des droits de l'enfant se lit comme suit :

\section{Principe 2}

L'enfant doit bénéficier d'une protection spéciale et se voir accorder des possibilités et des facilités par l'effet de la loi et par d'autres moyens, afin d'être en mesure de se développer d'une façon saine et normale sur le plan physique, intellectuel, moral, spirituel et social, dans des condítions de liberté et de dignité. Dans l'adoption de lois à cette fin, l'intérêt supérieur de l'enfant doit être la considération déterminante.

\section{Principe 3}

L'enfant a droit, dès sa naissance, à un nom et à une nationalité.

\section{Principe 4}

L'enfant doit bénéficier de la sécurité sociale. Il doit pouvoir grandir et se développer d'une façon saine : à cette fin, une aide et une protection spéciales doivent lui être assurées ainsi 
qu'à sa mère, notamment des soins prénatals et postnatals adéquats, l'enfant a droit à une alimentation, à un logement, à des loisirs et à des soins médicaux adéquats.

\section{Principe 5}

L'enfant physiquement, mentalement ou socialement désavantagé doit recevoir le traitement, l'éducation et les soins spéciaux que nécessite son état ou sa situation.

\section{Principe 6}

L'enfant, pour l'épanouissement harmonieux de sa personnalité, a besoin d'amour et de compréhension. Il doit, autant que possible, grandir sous la sauvegarde et sous la responsabilité de ses parents et en tout état de cause, dans une atmosphère d'affection et de sécurité morale et matérielle; l'enfant en bas âge ne doit pas, sauf circonstances exceptionnelles, être séparé de sa mère. La société et les pouvoirs publics ont le devoir de prendre un soin particulier des enfants sans famille ou de ceux qui n'ont pas de moyens d'existence suffisants. Il est souhaitable que soient accordées aux familles nombreuses des allocations de l'État ou autres pour l'entretien des enfants.

\section{Principe 7}

L'enfant a droit à une éducation qui doit être gratuite et obligatoire au moins aux niveaux élémentaires. Il doit bénéficier d'une éducation qui contribue à sa culture générale et lui permette, dans des conditions d'égalité de chances, de développer ses facultés, son jugement personnel et son sens des responsabilités morales et sociales, et de devenir un membre utile de la société.

L'intérêt supérieur de l'enfant doit être le guide de ceux qui ont la responsabilité de son éducation et de son orientation; cette responsabilité incombe en priorité à ses parents.

\section{Principe 10}

L'enfant doit être protégé contre les pratiques qui peuvent pousser à la discrimination raciale, à la discrimination religieuse ou à toute autre forme de discrimination. Il doit être élevé dans un esprit de compréhension, de tolérance, d'amitié entre les peuples, de paix et de fraternité universelle, et dans un sentiment qu'il lui appartient de consacrer son énergie et ses talents au service de ses semblables.

1387 (XIV). Publicité à donner à la Déclaration des droits de l'enfant

L'Assemblée générale,

Considérant que la Déclaration des droits de l'enfant invite les parents, les hommes et les femmes à titre individuel, ainsi 
que les organisations bénévoles, les autorités locales et les gouvernements nationaux à reconnaître les droits qu'elle énonce et à s'efforcer d'en assurer le respect.

1. Recommande aux gouvernements des États membres, aux institutions spécialisées intéressées et aux organisations non gouvernementales appropriées de donner la plus large publicité possible au texte de la déclaration des droits de Penfant :

2. Prie le Secrétaire général de donner à la déclaration une très large diffusion et à cette fin d'utiliser tous les moyens dont il dispose pour en faire publier et distribuer le texte dans toutes les langues possibles $\left(841^{\mathrm{e}}\right.$ séance plénière, 20 novembre 1959).

Ce qui ressort de la déclaration des droits de l'enfant, c'est qu'en principe le mineur doit bénéficier d'une «aide et d'une protection spéciale». Dans quelle mesure cet objectif est réalisé dans notre collectivité?

Comme nous l'avons indiqué plus haut quand une crise «se produit au sein de la famille et quand elle devient évidente, voire gênante ou nuisible» pour des tiers, les cours du bien-être-social jugent et statuent.

\section{LE SOUS-DÉVELOPPEMENT FAMILIAL ET LA MARGINALITÉ.}

Les enfants qui ont le triste privilège de bénéficier de cette forme de alargesse» collective se recrutent surtout parmi les «enfants de malheurn. Sur le plan socio-économique, ou sur le plan des relations affectives avec les adultes, ce sont des enfants pauvres! Il n'est pas inutile, à cet égard de consulter le rapport publié en mars 1975 par le Conseil national du bien-être-social, sous le titre "Les enfants pauvres».

On y lit, entre autre, qu'au Canada, de 6 millions 76 milles de moins de 16 ans, tels que recensés en 1971, 1 million 66 milles étaient pauvres. Dans le cadre du rapport on précise, en outre, le terme de la pauvreté de la façon suivante :

"Naître pauvre, c'est avoir toute votre vie une santé plus chancelante que celle des riches. Naître pauvre veut dire que vos chances de terminer votre éducation secondaire sont réduites et que la probabilité que vous fassiez des études universitaires est infime. Naître pauvre augmente vos chances qu'étant adolescent on vous jugera «délinquant» et qu'on vous mettra en «maison de correction"... Naître pauvre est injuste pour un enfant." 
Cette façon de souligner les conséquences d'un sous-développement familial qui généralement n'est pas uniquement économique, mais aussi socio-pédagogique, voire même psychique, demeure parfaitement conforme à la réalité. Il est impossible, en outre, "de traiter des problèmes des délinquants juvéniles et des formes de soins" qu'on leur inflige dans nos prisons, sans essayer de préciser au préalable qui ils sont et comment notre collectivité génère la délinquance juvénile. Cette approche est d'autant plus fondamentale, par ailleurs, que dans le grand public prévaut toujours l'attitude selon laquelle les mineurs condamnés par les Cours sont des "pas bons». Approche simpliste, certes, mais si profondement ancrée dans les esprits qu'on ne parvient pas à provoquer la moindre indignation tangible de l'opinion publique face au phénomène tel que l'emprisonnement des enfants.

Les mêmes groupes qui s'émeuvent quand on rapporte dans les media d'information la condamnation injuste d'un adulte, ou encore la mort d'un criminel poursuivi par la police, paraissent indifférents au fait que dans notre province des milliers de mineurs sont "traités" dans les prisons, parce que le ministère des Affaires sociales ne dispose pas d'un nombre suffisant d'établissements pour les recevoir...

Avant, toutefois, d'analyser la situation qui prévaut actuellement au Québec, il apparaît indispensable de situer le problème de l'enfance malheureuse dans laquelle se recrutent les déviants, dans sa dimension plus large. À cet égard, les données statistiques sont on ne peut plus révélatrices.

Quel lien existe-t-il entre la délinquance juvénile et la pauvreté? Abstraction faite de toutes les considérations philosophiques et socio-politiques, sur le plan strictement empirique, ce lien est triple; il comprend les problèmes de santé, de progression normale dans le cadre scolaire et de l'encadrement familial élémentaire.

En ce qui a trait aux problèmes de santé, le rapport préparé par Antoine Paquin et présenté au Colloque de la Commission des écoles catholiques de Montréal, le 18 avril 1970, indiquait que l'étude de 222 enfants de l'école Sainte-Catherine, a permis de dégager les conclusions suivantes :

a) $21,3 \%$ de ces enfants étaient mal nourris;

b) plus de $22 \%$ d'entre eux accusaient des retards de croissance aux points de vue de taille et de poids;

c) $27,5 \%$ manifestaient des retards du développement psycho-moteur. 
TABLEAU 2*

\begin{tabular}{lll}
\hline Provinces & $\begin{array}{l}\text { Nombre denfants } \\
\text { pauvres }\end{array}$ & $\begin{array}{l}\text { Pourcentages } \\
\text { du nombre total } \\
\text { denfants dans la } \\
\text { province }\end{array}$ \\
\hline Terre-Neuve & 92783 & 45,3 \\
ne-du-Prince-Édouard & 14111 & 37,3 \\
Nouvelle-Écosse & 75983 & 29,8 \\
Nouveau-Brunswick & 75520 & 34,9 \\
Québec & 528843 & 28,1 \\
Ontario & 400690 & 17,1 \\
Manitoba & 95874 & 31,6 \\
Saskatchewan & 114081 & 38,4 \\
Alberta & 135150 & 24,8 \\
Colombie-Britannique & 116301 & 17,9 \\
Yukon & 1160 & 17,8 \\
Territoires du Nord-ouest & 6489 & 42,4 \\
\hline
\end{tabular}

Le rapport ne précise pas combien de ces enfants ont été maltraités dans leur milieu familial, mais à cet égard il suffit de consulter les récentes déclarations des médecins de l'hôpital SainteJustine pour se rendre compte que le phénomène existe et qu'il est apparenté à celui du sous-développement socio-économique et socio-culturel de certains milieux familiaux.

En ce qui a trait aux problèmes de scolarisation, ils demeurent étroitement liés à ceux de santé. L'étude effectuée par Yvon Gauthier sur $\alpha$ L'activité fantasmatique d'enfants de milieux favorisé et défavorisén, indique ce qui suit :

Certains des enfants défavorisés abordent la classe de Petite Maternelle (4 ans) avec des ressources affectives intéressantes et leur développement demeure assez adéquat jusqu'en première année, mais... ils sont trop souvent sous l'empire d'un monde fantasmatique menaçant qui retarde le début de leur apprentissage scolaire et le rend problématique.

Cette constatation nous amène à l'analyse de la troisième dimension des causes qui forgent les liens entre la pauvreté et la délinquance juvénile, soit celle de l'encadrement familial élémentaire. Certes, il convient de préciser, à cet égard, que le manque d'encadrement familial élémentaire n'est pas l'apanage exclusif des milieux défavorisés, puisqu'il existe dans tous les milieux et a des origines diverses.

Il peut être provoqué, en effet, tout aussi bien par la rupture des liens maritaux des parents, que par l'incapacité du couple à

* «Les enfants pauvres», Rapport du Conseil national du bien-être social sur les enfants pauvres au Canada, mars 1975, page 5. 
assumer ses responsabilités à l'égard des enfants, ou encore par la démission consciente de certains intellectuels qui n'osent pas transmettre les valeurs éducatives élémentaires en raison de l'approche, on ne peut plus théorique, selon laquelle ces valeurs ne sont qu'une contrainte face à l'épanouissement naturel. À l'instar de Jean-Jacques Rousseau, mais sous la pression directe de certains "scientifiques» américains qui, après avoir fait fortune en divulguant des connaissances douteuses basées sur des expériences limitées, font désormais marche arrière, ces milieux familiaux appliquent, sans en être conscients, les théories décrites dans le célèbre traité sur l'éducation de l'Émile. Or, parmi les lecteurs et les admirateurs des théories de Jean-Jacques Rousseau, rares sont ceux qui savent qu'il avait eu une douzaine d'enfants illégitimes et légitimes et qu'ils furent tous placés par ses soins dans les crèches de l'époque et élevés aux frais de l'État...

Quoi qu'il en soit, le manque d'encadrement familial, n'est jamais stigmatisé avec la même rapidité quand il s'agit des milieux à l'aise, que quand le phénomène se produit chez les familles désavantagées. En effet, à cet égard, le lieu d'habitation et la situation du quartier jouent un rôle important. La vigilance de la police est plus présente et la stigmatisation des enfants plus grave dans les rues populeuses que dans les avenues ombragées de Westmount.

Le mineur qui vole l'argent dans le sac de sa mère, ou dans le portefeuille de son père, est inconnu de la police; celui par contre qui s'attaque aux biens d'un tiers est dénoncé généralement tôt ou tard.

Parallèlement, parmi les enfants des milieux sous-développés sur le plan socio-économique et affectif, que nous avons pu interroger, plusieurs nous ont déclaré : «Dès qu'il se passe quelque chose dans le quartier, les policiers viennent chez nous et m'amène au poste. Forcèment, ils me connaissent".

Ils ont fait des vols à l'étalage, ils ont fait des vols dans des appartements; elles ont essayé de gagner un peu d'argent en offrant leurs faveurs aux passants d'un certain âge...

Le code criminel définit les comportements de ce type sous des termes tels que : vol, vol avec effraction, recèle et «toute autre forme de vice..."

Et la stigmation ne s'arrête pas là... 
Dans les milieux familiaux qui offrent une certaine garantie de "respectabilité", la police et les Cours trouvent un "appui» sinon réel tout du moins théorique. Quand on parle de la justice pour riches et de l'injustice pour les autres, cela a des correspondances on ne peut plus précises en ce qui a trait aux mineurs. Ce n'est un secret pour personne que les écoles de protection disposent d'un nombre de places limité. Par conséquent, le juge des enfants dira volontiers à un pèré : monsieur, je retourne votre enfant à son foyer à charge pour vous de veiller à ce qu'il change de conduite...

Ả l'opposé, aucun juge ne peut prétendre en son âme et conscience, que s'il retourne le jeune dans un foyer où le père alcoolique, lui inflige systématiquement des "coups et sévices", tandis que la mère pratique ouvertement le plus vieux métier du monde, l'agent de probation parviendra quand même à compenser l'absence totale de l'encadrement familial...

En d'autres termes, tous les mineurs ont droit à la protection de la collectivité, mais en fait pour ceux qui n'ont pas d'encadrement familial cette "protection" est fréquemment une nunition imméritée qui se solde par la peine privative de liberté... Et, bien entendu, on joue sur les mots.

Un mineur issu d'un milieu criminogène peut être placé dans une école de protection sans avoir commis aucun délit; on dit alors que la collectivité lui assure la protection de la Cour. À l'opposé un jeune délinquant récidiviste peut rester avec la bénédiction du juge dans sa famille qui s'engage à le "protéger».

Le droit des enfants à un traitement équitable est faussé à la base par la simple et élémentaire exigence qui découle de leur minorité; il faut bien qu'ils vivent quelque part puisqu'on ne peut quand même pas les laisser se débrouiller seuls...

Ils ne sont ni "bons», ni "pas bons», ils sont différents des adultes, puisqu'ils ne possèdent rien en propre et que l'unique bien dont ils disposent, c'est-à-dire leur famille, leur échoit par le plus absolu des hasards, celui de leur naissance...

Analyser la situation des mineurs, en ce qui a trait à l'emprisonnement sans tenir compte de cette réalité fondamentale et commune à tous les jeunes, procède de la plus abjecte des hypocrisies! 


\section{L'EMPRISONNEMENT DES MINEURS ET LA LOI}

"On ne les met quand même pas en prison pour rien", affirment les gens bien intentionnés soucieux de préserver leur propre tranquilité et d'avoir en même temps bonne conscience.

Malheureusement, il est impossible d'avoir bonne conscience...

Selon la loi des jeunes délinquants, article 13 (1), p. 560 : Pendant qu'il attend son procés, en exécution de la présente loi, nul enfant ne doit être détenu dans une prison de comté ou autre, ni dans un autre lieu oủ des adultes sont ou peuvent être emprisonnés; mais il doit être gardé dans une maison de détention ou un refuge à l'usage exclusif des enfants, ou sous telle autre surveillance approuvée par le juge ou, en son absence par le shérif, ou, en l'absence du juge et du shérif, par le maire ou autre principal magistrat de la cité, ville, conté ou lieu.

Comme on le constate, le texte même de la loi semble imprécis, mais on peut toujours l'interpréter selon les circonstances.Ajoutons à cela que le niveau de discrétion des juges des enfants est beaucoup plus élevé que celui des juges des adultes. En effet, à la Cour du bien-être social, toute la procédure judiciaire demeure informelle.

Les journalistes ne peuvent pas assister aux audiences. Le mineur n'a pas, non plus, comme c'est le cas pour l'aduite, le droit formel de réclamer l'assistance d'un avocat. Il suffit donc qu'un juge décide que pour son bien et pour la protection de la société, il est préférable qu'il soit emprisonné, et il le sera.

Parallèlement, au point de vue des structures, la situation demeure complexe, pour ne pas dire confuse. Les juges relèvent du ministère de la Justice et les prisons aussi. Les écoles de protection, par contre, ont une autonomie en ce qui a trait à la réception des cas et elles relèvent toutes du ministères des Affaires sociales.

En d'autres termes, la sentence du juge des enfants, contrairement à tous les autres juges, n'est pas exécutoire, sauf s'il condamne le mineur à la prison. En effet, les centres de détention sont des endroits qui ne jouissent d'aucune autonomie. Un directeur de prison qui refuse de recevoir un mineur peut être accusé de mépris de cour et dans tous les cas démis de ses fonctions. Le directeur d'une école de protection par contre, peut invoquer l'incapacité 
de «traiter» un mineur envoyé dans son institution par le juge, en raison du programme en vigueur, du manque d'espace, ou encore de la non-disponibilité du personnel qui a organisé des «journées d'études».

Pour comprendre pourquoi les mineurs se retrouvent en prison, il ne s'agit pas, en somme, d'analyser les taux de la criminalité des jeunes et leurs variables, mais d'additionner le nombre de places disponibles dans les centres d'accueil et dans les écoles de protection et d'établir un parallèle entre ce total et celui des jeunes qui passent devant les Cours du bien-être social...

Précisons, toutefois, qu'il ne s'agit pas là d'une critique du principe de l'autonomie des établissements de traitement des mineurs, puisqu'elle est indispensable pour maintenir un certain standard de ce traitement, mais plutôt d'une constatation globale relative à l'insuffisance de structures, telles qu'elles' existent. Ajoutons qu'il n'est guère rentable sur le plan électoral, pour un ministre, d'annoncer qu'il va investir une partie de son budget, aussi minime puisse-t-elle être, pour assurer aux mineurs ayant besoin de protection un traitement équitable et cela suffira pour expliquer les statistiques officielles de détention carcérale des enfants...

TABLEAU 3

Mineurs incarcérés dans les prisons du Québec

\begin{tabular}{llccc}
\hline \multicolumn{4}{c}{ Garçons et filles (1970-1974) } \\
\hline \multirow{4}{*}{1970} & Moins de 16 ans & 16 à 18 ans & Total \\
& Orévenus et détenus & 1217 & 2149 & 3366 \\
1971 & Ont purgé une sentence & 20 & 137 & 157 \\
& Prévenus et détenus. & 597 & 1467 & 2064 \\
1972 & Ont purgé une sentence & 3 & 83 & 86 \\
& Prévenus et détenus. & 354 & 970 & 1324 \\
1973 & Ont purgé une sentence & 0 & 59 & 1139 \\
& Prévenus et détenus. & 302 & 837 & 1139 \\
1974 & Ont purgé uné sentence & 2 & 62 & 64 \\
& Prévenus et détenus. & 323 & 984 & 1307 \\
& Ont purgé une sentence & 2 & 72 & 74 \\
\hline
\end{tabular}

\section{E. LES JEUNES DANS LES PRISONS POUR ADULTES}

C'est ainsi que selon le Rapport annuel de la direction générale de la probation et des établissements de détention du ministère de la Justice, pour 1974, on rapporte les relevés suivants :

Le tableau ci-dessus est on ne peut plus révelateur. Il n'est 
guère besoin en effet, d'être un spécialiste pour constater la surprenante irresponsabilité de notre collectivité. On sait que les prévenus, ce sont des personnes qui attendent la décision judiciaire, tandis que les gens qui ont purgé une sentence, ce sont ceux qui ont été dûment condamnés. Par conséquent, selon les statistiques de nos prisons, les prévenus de moins de seize ans et de moins de dix-huit ans constituent la majorité des mineurs incarcérés. En soi, c'est déjà un scandale! Plus encore, on peut considérer que nous emprisonnons illégalement des mineurs, puisque selon le Code criminel (article 13, (1), p. 560), cette procédure ne se justifie pas.

Or, il est difficile de concevoir qu'à notre époque, nos gouvernements ne parviennent toujours pas à assurer à quelque mille trois cent jeunes un autre moyen de traitement que celui à l'intérieur des murs d'une prison pour adultes. Parallèlement, il est absolument impensable de considérer qu'on puisse user encore d'une hypocrisie sociale suffisante pour prétendre que la prison puisse être l'unique «lieu où l'enfant peut être détenu en sûreté»... (art. 13 par. 4).

Est-il nécessaire de rappeler à ce propos que les bibliothèques spécialisées sont remplies de volumes où des théoriciens et des praticiens démontrent les effets néfastes de la sous-culture carcérale? Est-il indispensable d'affirmer une fois de plus que l'incarcération qui peut être une nécessité dans le cas d'un adulte, ne saurait être considérée comme telle quand il s'agit d'un groupe restreint des mineurs qui peuvent dans tous les cas, même ceux de meurtriers, être traités de façon plus conforme à leurs droits élémentaires de mineurs que la société se doit, en principe, de protéger?

Précisons enfin que la relation entre le nombre de mineurs qui ont purgé une sentence et celui des jeunes incarcérés comme prévenus permet d'indiquer clairement que ce ne sont pas les juges qui doivent être considérés comme incapables de respecter les droits des mineurs à un traitement autre que celui des adultes. Il en ressort, au contraire, que ce sont surtout les gouvernements provinciaux successifs qui semblent incapables d'assumer leurs responsabilités à l'égard des jeunes ayant des comportements marginaux.

À l'intérieur du gouvernement, deux ministère sont directement impliqués; celui de la Justice et celui des Affaires sociales. En ce qui a trait au ministère de la Justice, il n'est pas inutile de citer l'extrait du rapport annuel de la Direction générale de la probation et des établissements de détention, où on lit, en page 124 , ce qui suit : 
Le problème de l'incarcération des juvéniles dans des établissements pour adultes, que nous voyions avec satisfaction diminuer en intensité au cours des dernières années et soudainement revenu à la surface avec plus de force que jamais au cours des derniers mois de l'année 1974.

Le tout a débuté avec les troubles qui ont surgi au Centre Berthelet de Montréal au mois de novembre. $\AA$ cause de la situation explosive qui prévalait à cet endroit, nous nous sommes vus dans l'obligation, à la demande du ministère des Affaires sociales, d'héberger quatre-vingt-neuf (89) jeunes de 14 à 18 ans. Les jeunes furent dirigés vers les établissements de St-Hyacinthe et de Cowansville qui, pour la circonstance, avaient transféré ailleurs les prévenus et détenus adultes. Joliette, des locaux séparés de ceux des adultes furent aménagés pour les nouveaux arrivants.

Une attention particulière fut accordée aux jeunes. Dès leur arrivée, les préposés en service social les rencontrèrent afin de détecter les besoins de chacun et d'y apporter une solution immédiate.

Le contact avec leur milieu familial ou social immédiat fut rétabli dès l'entrée. De plus, de concert avec le ministère des Affaires sociales, nous pouvions compter sur les services d'une équipe multidisciplinaire de spécialistes en sciences humaines dans les cas des jeunes qui présentaient des problèmes émotifs et dont l'état mental ou physique méritait une attention immédiate. Dans la mesure de nos moyens, nous avons cherché à multiplier les formes d'activités occupationnelles et récréatives.

À Cowansville, en particulier, grâce à la collaboration d'un comité de citoyens, les jeunes ont eu l'avantage de bénéficier de visites de l'extérieur, de projection de films et d'un programme de loisirs préparé à leur intention.

Cette détention massive de juvéniles en milieu adulte, qui s'étendit sur une période de deux (2) semaines, trouvait son explication dans la situation pénible que vivaient les autorités du Centre Berthelet. Elle constituait une opération de dépannage et n'en représente pas moins une expérience que nous ne désirons plus revivre. Le ministère des Affaires sociales partage probablement encore plus que nous cette conviction. En ce qui nous concerne, nous souhaitons qu'une solution définitive soit trouvée dans les meilleurs délais au problème de la détention des jeunes au Québec.

Comme on le constate sur la base de ce texte, le ministère de la Justice ne semble pas intéressé à recevoir des mineurs dans les prisons pour adultes, mais il apparaît malheureusement que le ministère des Affaires sociales n'a pas du tout l'intention d'assumer ses responsabilités. 
À cet égard une analyse succincte du Rapport annuel du ministère des Affaires sociales demeure on ne peut plus instructive. En premier lieu, dans le rapport pour 1971-1972, présenté par Claude Castonguay, ministre de l'époque, on consacre à peine quelques lignes aux problèmes des mineurs délinquants.

Dans le rapport pour 1972-1973, qui compte 186 pages au total, on relève par contre des déclarations d'intention, dont en page 113, la phrase suivante qui a trait aux objectifs pour l'année 1972 : «Le ministère des Affaires sociales entreprendra la révision des politiques concernant l'enfance délinquante et l'établissement d'un nouveau partage des responsabilités avec le ministère de la Justice."

Le rapprochement entre ce minuscule passage et le texte du rapport du ministère de la Justice publié en 1974, est fort intéressant. En ce qui a trait aux statistiques, en 1972 un nombre total de 1383 mineurs ont eu le douteux droit d'être détenus dans les prisons du Québec...

Plus encore, les ministres changent, mais les politiques, ou plutôt l'absence d'une politique de protection des droits des mineurs, demeurent.

Le rapport annuel du ministère des Affaires sociales pour 1973-1974 comporte très précisément deux pages, sur 60 qu'on peut considérer comme vaguement consacrées aux problèmes des droits des mineurs à la protection de la collectivité. Les statistiques des institutions destinées à recevoir les jeunes marginaux et les relevés budgétaires indiquant les sommes qu'on leur consacre, sont remplacées par quelques déclarations de bonnes intentions et par des projets de restructuration dont il est pratiquement impossible d'évaluer la réalisation et à plus forte raison l'efficacité probable. Il n'est nullement question dans ce rapport, des droits des mineurs de ne pas aller en prison, mais on y distille goutte à goutte un paternalisme digne du tournant du siècle.

\section{F. LES DROITS DES MINEURS ET LES PERSPECTIVES D'AVENIR}

Qu'est-ce qui ressort de tout cela?

Que les mineurs n'ont pas en fait de droits, mais uniquement des devoirs? 
Leur statut est inhérent à la condition même d'une personne humaine dépendante par définition, compte tenu de sa minorité.

En principe, cette dépendance doit s'accompagner d'une responsabilité des adultes non seulement familiale, ou individuelle, mais collective. Or, au niveau de la collectivité, et cela est vrai non seulement pour le Québec, mais pour la plupart des sociétés occidentales, les responsabilités, sont assumées surtout à travers la famille. Cela signifie que quand la famille parvient à remplir ses fonctions d'encadrement de façon qui élimine l'intervention de l'appareil de justice, la collectivité fournit les services médicaux et les services scolaires. Le mineur a l'obligation d'obéir et d'accepter d'être soigné et d'être éduqué.

Dès le moment, cependant, où l'écran protecteur de la famille s'avère déficient, ou insuffisant, la société commence à se protéger contre les agissements dits marginaux. Le traitement en cages, ou l'emprisonnement, est inefficace à long terme, comme cela a été prouvé à maintes reprises dans le cadre de diverses recherches sur la récidive; peu importe! Faute d'un équipement institutionnel suffisant, on reçoit dans les prisons pour adultes, les prévenus et les détenus de moins de seize ans, dangereux ou pas.

Cette situation de fait a été dénoncée au Québec à maintes reprises, autant dans les rapports des commissions d'enquête dont celle présidée par le juge Yves Prévost, que dans les travaux effectués par des universitaires. On a proposé des réformes et on a formulé des recommandations...

Quelles sont les perspectives de leur réalisation?

\section{G. LE LIVRE BLANC DU MINISTĖRE DE LA JUSTICE}

Le Livre Blanc du ministère de la Justice, publié en 1975, devait theoriquement répondre à cette question, mais malheureusement c'est un texte très incomplet. Certes, ce volume de 360 pages demeure un ouvrage de référence fondamental, ne serait-ce qu'en raison de la clarté de son style et des relevés statistiques dont la précision permet de le considérer comme un document de base pour les réformes à venir, il n'en reste pas moins qu'à tort ou à raison on s'attendait à autre chose...

On espérait qu'après des années d'études et d'analyses faites en vue de réformes des législations fédérale et provinciale de la 
justice des mineurs, le Livre Blanc définira enfin des objectifs clairs d'une nouvelle philosophie respectueuse des droits des mineurs. Le chapitre III du Livre Blanc, intitulé «La question du tribunal de la famille et la protection de la jeunessen, demeure néanmoins la preuve d'un conformisme et d'un défaitisme plutôt surprenants. Le chapitre comporte 24 pages qu'il faut étudier en détails pour comprendre pourquoi dans notre province on reçoit encore, plus de 1300 enfants dans les prisons pour adultes. En premier lieu, on traite du problème de la création du Tribunal de la famille, en citant des extraits du rapport de l'Office de révision du code civil qui recommande l'institution «d'un tribunal de la famille doté d'une juridiction intégrée sur l'ensemble des matières familiales». Dans le Livre Blanc, on conclut toutefois, que "pour des motifs constitutionnels, il ne semble pas possible de créer un tribunal de la famille, autonome et distinct, ayant toute la compétence civile criminelle et pénale en matière de droit familialı.

Or, comme c'est le cas en Colombie-britannique, on peut fort bien établir des modes de procédure permettant de contourner les difficultés dont on traite dans le Livre Blanc. Le juge Robert Sauvé, président de la Commission des Services juridiques, suggère par exemple, à ce propos qu'il suffit de conclure des ententes informelles entre les cours en commençant l'expérience dans quelques districts judiciaires pour l'élargir ensuite à tous les autres.

Parallèlement, actuellement, la Cour du bien-être social défère chaque année un certain nombre de cas de jeunes délinquants aux tribunaux criminels ordinaires. Le total de ces mineurs représente au-delà de trois cents pour 1973, en ce qui a trait à Montréal uniquement. Ces jeunes, à cause de la gravité de leurs délits, ou de la récidive, sont généralement condamnés à la prison... C'est là une façon pour les juges des enfants de se libérer de l'odieux d'une condamnation, parce qu'en fait, ils disposent des pouvoirs suffisants pour assumer cette responsabilité...

Tout en demandant la spécialisation des juges des Cours du bien-être social, tout en insistant sur l'importance de la relation humaine et sociale qui devrait s'établir entre le juge et le mineur en vue de son "traitement" et non pas de sa "punition", on ne semble pas prêt à régler le problème des cas déférés. En page 142 du Livre Blanc on recommande même à cet égard deux approches contradictoires. 
En effet, au paragraphe 3.3, on lit : "Que généralement l'on maintienne les compétences actuelles de la Cour supérieure et de la Cour du bien-être social, ou de la Cour du Québec, section de la famille et la jeunesse, mais que l'on étudie un réaménagement de celles-ci afin d'éviter des conflits de juridiction." Au paragraphe suivant 3.4 , on recommande néanmoins «que l'on confie à la Cour du Québec, section famille et jeunesse, toutes les matières criminelles qui concernent les époux, les parents et les enfants, sauf le loisir pour la Cour de remettre aux tribunaux criminels ordinaires des causes particulières lorsque cela est jugé opportun". En réalité, il ne s'agit pas là du conflit de juridictions, mais surtout du conflit entre la philosophie d'aide et de protection que la collectivité doit assumer à l'égard des mineurs et la conception strictement répressive de la délinquance juvénile, exercée au mépris des droits des jeunes.

Les contraintes constitutionnelles sont utilisées également pour atténuer notre responsabilité collective dans le domaine de la défense des droits des mineurs. C'est ainsi que tout en reconnaissant dans le Livre Blanc la nécessité de réformes, on affirme que "ces réformes mettent en cause l'autorité fédérale qui a compétence en matière de droit criminel et, par conséquent, en matière de délinquance juvénile, et l'autorité provinciale qui a compétence en matière de protection de la jeunesse, notamment à l'égard des enfants en état de danger physique ou moral, ou dont le comportement est qualifié de prédélinquant».

Or, les définitions en cette matière étant en fait une porte ouverte à une autonomie très étendue des juges des enfants, il s'agit d'une hypocrisie consacrée par nos législations. Dans tous les cas des délits, les juges peuvent en effet décider que le mineur «est en danger physique et moral" et l'entendre en vertu de la Loi de protection de l'enfance et de la jeunesse et non pas de la Loi sur les jeunes délinquants.

En d'autres termes, contrairement à ce qui existe quand is s'agit des adultes, les juges des enfants ne sont jamais tenus d'appliquer le code criminel, de condamner un mineur à la détention dans une prison pour adultes, ni à plus forte raison de signer le mandat de détention préventive. Plus encore, sur le plan strictement légal, toute détention préventive d'un mineur est une injustice flagrante, puisqu'il n'est généralement pas défendu par un avocat, comme c'est le cas pour un adulte, et ne peut être libéré, comme c'est possible même pour des criminels tristements célèbres, sur cautionnement ou sur parole. 
Selon certains, les juges des enfants se laissent influencer par les rapports de la police...

Voici une autre excuse qui justifie le paternalisme de notre société. Car en fait, et c'est là une des lacunes du chapitre III du Livre Blanc, de ne pas le souligner, le premier contact qui s'établit entre le jeune délinquant et les organes de contrôle, n'est pas celui avec la Cour, mais avec la police. Plus encore, c'est de l'attitude des policiers que dépend fréquemment le sort que subira le mineur et même son éventuelle incarcération. Compte tenu du fait qu'en Grande-Bretange, le rôle de la police auprès des mineurs délinquants, ou prédélinquants, est reconnu comme particulièrement "social», par opposition à celui beaucoup plus répressif qui prévaut ailleurs, on a fait venir au Québec, il y a quelques années, des missions spéciales de policiers britanniques. Ils étaient chargés de rencontrer les policiers de Montréal et d'expliquer en détails, la façon selon laquelle ils procèdent. Depuis, on a créé une unité de police dont les agents reçoivent une formation spéciale et doivent s'occuper uniquement des mineurs. Cette unité comprend à l'heure actuelle deux cents hommes dont une partie est affectée aux divers postes. Étant donné, cependant, que les effectifs sont insuffisants, compte tenu des besoins de la population de Montréal, que les distances à parcourir sont trop grandes et que le commandement n'est pas unifié, l'unité ne parvient pas à répondre à la demande. Les policiers affectés aux postes, dépendent des commandants des postes et non pas du commandement de l'unité, ils sont donc obligés fréquemment de remplir des tâches autres que celles auprès des mineurs. Une unité de deux cents hommes, des structures qui ne correspondent pas aux besoins : un autre exemple de notre irresponsabilité collective à l'égard des mineurs...

Et il est inutile d'espérer que cela va changer. On prétend, parât-il, en haut lieu, que le développement de l'Aide jeunesse risque de provoquer une mauvaise publicité pour nos policiers. Le public serait porté, semble-t-il, à distinguer entre les «bons policiers» de l'Aide jeunesse, et les autres...

Le Livre Blanc passe sous silence le rôle de la police spécialisée, aussi fondamental puisse-t-il être, mais traite par contre en détails de la philosophie préventive. On y lit à ce propos le passage suivant, en page 145:

En ce qui concerne le domaine de la protection de la jeunesse, de la prévention et de la sanction de la délinquance chez 
les juvéniles, cette mise au point des lois et des institutions a la plus haute importance pour les enfants et les adolescents en proie à des problèmes de comportement, pour leur avenir et leur intégration harmonieuse éventuelle en société. En effet, les statistiques en matière de criminalité démontrent que c'est à l'âge de 18 ans que le comportement criminel ou associal atteint son maximum. Il est également démontré que le comportement criminel chez l'adulte est une habitude acquise ou apprise dans l'adolescence et même l'enfance. De ces données, il faut conclure que des interventions appropriées au cours des années de développement chez l'enfant ou l'adolescent peuvent représenter la différence entre le succès ou la catastrophe d'une vie. Les effets d'intervention fructueuse à l'âge de la formation sont donc incalculables tant en ce qui concerne l'adolescent ou l'enfant lui-même que la socièté en général.

Traiter «d'intervention fructueuse à l'âge de la formation", c'est faire preuve d'hypocrisie. En effet, dans le contexte québecois, l'intervention est tout simplement impossible, aussi longtemps que ne se produit pas une «crise». Étant donné le respect de l'autorité parentale et les droits de la famille, étant donné l'absence totale des contrôles sociaux préventifs, autant au moment de la naissance qu'à travers le cycle préscolaire et scolaire, nous n'intervenons que lorsqu'il est déjà «trop tard n. Plus concrètement le mineur maltraité, brutalisé, ou négligé par ses parents, n'est sujet à une protection sociale quelconque qu'au moment où les conséquences des conditions dans lesquelles il est obligé de vivre, dérangent la collectivité. Dans ce contexte, il peut s'agir aussi bien d'un enfant qu'on amène à l'hopital mutilé, que d'un adolescent qui a commis un délit pour échapper à la "sollicitude» familiale, ou d'une jeune fille victime d'inceste qui décide de chercher des partenaires autres que son père...

Les développements qui suivent dans le Livre Blanc, les déclarations d'intentions précitées prennent dès lors d'autant plus de relief.

"En matière de protection de la jeunesse et de délinquance juvénile, nous devons constater que les attitudes des personnes qui y cuvrent se partagent entre deux écoles principales : ceux qui ont comme préoccupation l'utilisation de schémas juridiques par l'action des tribunaux, et ceux qui préconisent des méthodes à caractère social par l'action d'organismes qui se situent en dehors du circuit judiciaire." Il n'y est question nullement cependant des contrôles préventifs, des examens médicaux obligatoires suivis d'une 
assistance sociale préventive, ou encore de certaines formes de tutelles partielles auprès des familles - problèmes, en vue de protection de l'enfance.

Il n'en reste pas moins qu'en termes de «traitement", les auteurs du Livre Blanc formulent des recommandations intéressantes et très positives en proposant un traitement social par opposition au schéma judiciaire; ce qui a été consacré d'ailleurs dans le projet de la nouvelle loi sur la protection de la jeunesse au Québec, que nous analysons sommairement dans les pages qui suivent :

C'est ainsi qu'on y lit en page 149 :

Nous recommandons :

3.10 QUE le gouvernement institue une Commission de la protection de la jeunesse, dont les membres seront désignés sur la recommandation des ministres de la Justice et des Affaires sociales;

3.11 QUE cette commission assure les fonctions de coordination entre la Cour de bien-être social, les C.S.S. et les institutions qui accueillent les jeunes et que cet organisme se voit confier des fonctions de prévention et d'éducation dans le domaine de la jeunesse;

3.12 QUE cette commission soit chargée de former et de reconnaître des comités au niveau local ou régional;

3.13 QUE ces comités aient pour fonction de faire le tri des cas d'enfants devant être soumis à un traitement social ou judiciaire, suivant les cas, et que ces comités soient habilités à exercer une action de protection générale à l'égard de la jeunesse dans leur territoire.

Le responsable de ces comités devrait pouvoir décider qu'un enfant, qu'il s'agisse d'un cas de délinquance ou de protection, ne soit pas traduit devant la Cour de bien-être social dans les cas qui ne requièrent pas véritablement une intervention judiciaire.

"Nous favorisons donc, écrivent les auteurs du Livre Blanc, que l'on retienne le critère proposé par le livre blanc britannique Children in trouble, à savoir que les enfants et adolescents ne doivent être traduits dans le système judiciaire que si cela est souhaitable et bénéfique pour l'enfant et la société, tout en maintenant au représentant du ministère de la Justice une opinion prépondérante dans les cas graves." 
Comme nous préconisons cette approche depuis 1969 déjà', soit depuis l'époque de la Commission Prévost et comme nous avons préparé des rapports de plus de 1000 pages en citant les cadres britannique et suédois pour mieux documenter notre argumentation, nous ne pouvons que considérer comme excellente, la décision du ministère de la Justice de s'orienter dans cette voie. Les législations sont cependant ce qu'elles sont et tout dépend de leur application et de leur mise en pratique.

C'est ainsi que sur le plan législatif, par exemple, les mineurs auront le droit d'être défendus par des avocats, mais on ne précise toujours pas qui l'avocat devra représenter dans les cas des conflits entre les parents et l'enfant, selon la nouvelle législation. Plus concrètement quand les parents, qui n'ont pas assuré à l'enfant un encadrement suffisant, supplient le juge de, "l'enfermer", devrat-on disposer de deux défenseurs légaux et d'un procureur de la Couronne qui verra à intervenir au nom de l'intérêt de la collectivité?

On est en droit, nous semble-t-il, de s'étonner qu'on ne précise pas que les avocats d'aide juridique doivent défendre uniquement les droits des enfants, et cela tout autant face aux adultes qui en ont la charge que face à la collectivité en tant que telle...

Parallèlement on refuse d'admettre les représentants des media d'information aux séances de la cour. La présence des journalistes aux procès des adultes est considérée comme une garantie additionnelle de l'équité de la justice, mais un mineur peut être condamné à la prison pour adultes sans que l'opinion publique en soit informée...

Certes, on recommande, dans le Livre Blanc, en page 15, paragraphe 3.16 «que les membres et représentants de groupements bénévoles ou à caractère social reconnus par le juge en chef, soient admis aux séances de la courn, mais cette proposition ne répond pas aux mêmes objectifs.

N'est-ce pas paternaliste de permettre que le juge en chef décide quel groupement peut être admis aux séances et cela d'autant

I. A. Parizeau : Etude comparative sur la délinquance juvénile en Suède, en France, en Angleterre et au Québec, rapport publié par la Commission d'enquête sur l'administration de la justice en matière criminelle et pénale au Québec, 1970, vol. IV, t. 2, 381 pages: vol. IV, t. 3, (378 pages): A. Parizeau (avec D. Szabo et D. Gagné) : Face à face : l'adolescent et la société, Bruxelies, Charles Dessart, 1972, (332 pages). 
plus qu'une procédure de ce type exclu à priori tout appel à l'opinion publique?

Il est évident, en effet, que le groupement en question se gardera bien de dénoncer éventuellement une injustice commise à l'égard d'un mineur sachant qu'une telle action est susceptible de le priver de toute intervention ultérieure.

À l'heure où les séances de la Commission sur le crime organisé sont télévisées et radiodiffusées, malgré le préjudice que cela peut occasionner à certains témoins, la justice des mineurs se cache... Pourquoi? Pour assurer un surcroît de pouvoir au juge en chef des Cours du bien-être social?

\section{H. LES NOUVELLES LÉGISLATIONS ET LA DÉTENTION DES MINEURS}

À la suite de la publication par le ministre de la Justice du Livre Blanc, deux nouveaux projets de Loi concernant les mineurs ont été présentés; dont le premier au niveau du gouvernement fédéral et le deuxième à celui de la législature provinciale.

C'est ainsi que le 27 juin 1975, M. Claude E. Forget, ministre des Affaires sociales de la province de Québec déposait devant l'Assemblée nationale l'avant-projet de la Loi sur la protection de la jeunesse, destinée à remplacer la loi en vigueur (S.R. 1964, chapitre 220). Une analyse rapide de ce projet suffit pour constater que le dialogue de sourds engagé entre le ministre de la Justice et le ministère des Affaires sociales continue...

Certes, on y reprend les propositions relatives aux comités, déjà formulées dans le Livre Blanc, mais on a nettement l'impression que le ministère des Affaires sociales veut bien accepter que les travailleurs sociaux s'occupent des enfants âgés de moins de quatorze ans, mais pas au-delà de cette limite.

C'est ainsi qu'on y lit en page 54 :

Si toutefois la Cour en vient à la conclusion qu'un enfant âgé de 14 ans ou plus :

a) a commis un crime qui, s'il avait été commis par un adulte, aurait pu entraîner 3 années de détention ou plus; ou

b) a été si souvent trouvé coupable par la Cour pour infractions à une loi, ou un règlement que les moyens ordinaires 
de protection doivent être considérés comme ne pouvant plus lui être applicables de façon efficace;

la Cour peut ordonner son hébergement obligatoire dans un établissement de détention au sens de la Loi de la protection et des établissements de détention (1969, chapitre 21 ) pour la période qu'elle fixe.

En langage clair, cela signifie qu'en pratique quand les comités ne voudront plus s'occuper d'un cas particulièrement difficile, ils n'auront qu'à «l'abandonner», à ces messieurs les juges pour qu'ils assument l'odieux d'une condamnation à l'emprisonnement. Connaissant les difficultés chroniques que les travailleurs sociaux rencontrent quand il s'agit de placement du mineur en dehors de son milieu familial, faute de disponibilités autant en ce qui a trait aux foyers de substitution (foyers nourriciers) que les groupes de vie, ou les écoles de protection, y compris même les hôpitaux, quand un traitement prolongé s'impose, psychiatrique, ou de désintoxication, on se rend compte immédiatement de la «tentation» que ce court texte présente. La question qui se pose est celle de savoir pourquoi les juges des enfants ne protestent pas, enfin, et ne décident pas de refuser de siéger sur le banc de la Cour du bien-être Social. D'une étape à l'autre leurs responsabilités deviennent de plus en plus lourdes et leurs disponibilités s'amenuisent comme une peau de chagrin.

En fait si le projet de Loi sur la protection de la jeunesse devait être adopté tel quel, la principale fonction de nos juges des enfants sera de condamner à la prison les mineurs âgés de plus ou de moins de 14 ans, que les comités des travailleurs sociaux leur abandonneront en toute magnanimité.

Ả cet égard le «Rapport du Comité du ministère du Solliciteur général sur les propositions formulées en remplacement de la Loi sur les Jeunes délinquants" tel que soumis au Solliciteur général du Canada, en 1975, est également très révélateur.

Dans les recommandations, le Comité établit une dichotomie entre le terme de l'"enfant», qui «désigne une personne apparemment ou effectivement âgée de moins de quatorze ans" et celui de «jeune» qui «désigne une personne apparemment ou effectivement âgée de plus de quatorze ans et de moins de dix-huit ans...». C'est là une amélioration incontestable par rapport à la loi en vigueur selon laquelle il n'y avait qu'une seule catégorie de personnes responsables dès l'âge de 7 ans et jusqu'à une limite de 16, 17 ou 18 ans, fixée selon la décision des provinces. 
Il n'en reste pas moins qu'on y lit en page 103, ce qui suit : 660.1 (1) Lorsqu'un tribunal condamne une personne âgée de moins de dix-huit ans à une peine d'emprisonnement, cette personne peut, sous réserve du consentement du directeur provincial, être détenue sous la garde d'un milieu fermé, tel que le définit la Loi sur les jeunes qui ont des démêlés avec la justice, pour purger en tout ou en partie, sa sentence, et ce jusqu'à ce qu'il ait atteint l'âge de 21 ans; cependant, si la personne qui a la charge de ce lieu atteste auparavant que, compte tenu de la mauvaise conduite du jeune, celui-ci ne peut être détenu plus longtemps dans ce lieu sans risque sérieux qu'il ne s'évade ou que cela soit préjudiciable à la réadaptation des autres personnes détenues dans ce lieu, il peut, dans l'un et l'autre cas, être emprisonné pour le reste de la durée de sa sentence dans tout lieu où il aurait ordinairement pu être emprisonné n'eût été le présent article.n

En d'autres termes, une fois de plus le législateur fédéral, comme le législateur provincial acceptent non seulement des exceptions, mais d'une façon globale reconnaissent que si les institutions pour mineurs veulent se débarrasser d'un élève «âgé de moins de dix-huit ans», la prison sera toujours là pour leur servir de dépotoir.

Pour faire un parallèle, ad absurdum, si on permettait aux enseignants débordés, surmenés et faisant quotidiennement face à des groupes d'adolescents, parfois fort difficiles à contrôler, de se débarrasser des têtes fortes en les acheminant vers des «institutions spécialisées», il y aurait des probabilités marquées qu'ils profitent d'une pareille solution.

A plus forte raison dans les écoles de protection, où la mission des éducateurs n'est guère aisée et ou en plus les élèves ne sont pas protégés par les familles prêtes à intervenir en leur faveur, comment concevoir qu'ils ne s'empressent pas, à l'occasion, d'expédier en prison un garçon de moins de 14 ans ou plus, peu importe, parce qu'il perturbe tout le groupe, commet des actes de violence et casse les vitres.

En ce qui a trait aux «risques sérieux qu'il ne s'évade» dont on traite dans ce paragraphe du projet de loi, tous les praticiens savent que ce risque est non seulement sérieux, mais même souvent mis en exécution, dans toutes les écoles de protection du Québec et du Canada...

Après tout, le goût d'évasion est propre à la jeunesse y compris les «enfants du malheur»... 
Espérer que l'intervention juste et intelligente des juges, va tempérer les risques de l'imprécision de la loi, c'est faire preuve d'un optimisme tout à fait injustifiable. Face aux administrateurs de l'institution qui refusent de continuer à s'occuper d'un cas, le juge des enfants ne peut qu'essayer de discuter, mais en fin de compte il est bien obligé de céder, en vertu du principe élémentaire que l'adolescent doit être placé quelque part, dans un lieu de vie et de formation... La prison l'attend, en somme, toutes portes ouvertes.

En dernière analyse, mentionnons que le fait d'être envoyé à la prison n'est guère un argument éducatif puissant. Selon l'expérience que nous avons acquise en interrogeant certains jeunes, ils préfèrent une cellule de prison où on ne fait rien, où on fume et où on écoute la radio, à l'école de protection, où quand même certains réglements obligent à s'acquitter d'une tâche ou à suivre un enseignement.

Il faut quand même aider les écoles de protection à maintenir un régime de vie, objectera-t-on.

Certes, mais alors la loi doit préciser que la détention ne peut s'appliquer qu'aux jeunes de plus de quatorze ans et pour des périodes très courtes (une semaine au minimum à deux semaines au maximum, par exemple), et uniquement dans les cas marginaux où il a été démontré, à la satisfaction des comités des travailleurs sociaux impliqués et du juge, qu'aucune autre solution n'est possible.

Il est étrange de constater que, malgré les tristes expériences des dernières années où une grève à Berthelet remplissait de jeunes les prisons pour adultes et où le ministère de la Justice du Québec considérait comme utile de le déplorer dans son Livre Blanc, les législateurs fédéraux continuent à maintenir des projets de législation ouvrant largement la porte à toutes les faiblesses des structures de traitement des mineurs marginaux.

En pratique, cela signifie que les institutions menacées, par exemple, par la révision de leurs normes, pourront être paralysées à cause des journées d'étude de leur personnel et les prisons pour adultes, comme cela s'est déjà fait récemment, vont évacuer leurs "pensionnaires», criminels endurcis, ou pas, pour accueillir ceux dont le principal drame consiste à ne pas avoir de familles pour les recevoir... 
Peut-on parler de justice face aux semblables situations concrètes?

\section{L'EMPRISONNEMENT DES MINEURS ET LES CONFLITS INTERMINISTÉRIELS}

En d'autres termes, l'analyse rapide des projets de législations; fédérale et celle de la loi sur la protection de la jeunesse de la province de Québec permet de faire trois principales constatations.

En premier lieu, les structures décisionnelles deviennent sociales après avoir été surtout judiciaires, ce qui constitue, selon nous, un progrès incontestable. En deuxième lieu, les autorités judiciaires conservent toutes les responsabilités et toutes les prérogatives en ce qui a trait aux condamnations à la détention.

En troisième lieu, les travailleurs sociaux qui œuvrent dans le champ disposent des pouvoirs, mais leurs responsabilités ne sont pas délimitées clairement, ce qui leur permet de transférer tous les cas qu'ils considèrent comme difficiles au système judiciaire proprement dit.

C'est à ce niveau que se situe le problème de mise en pratique des législations proposées et il est d'autant plus grave qu'il est impossible de faire abstraction de l'éternel conflit qui existe entre les ministères de la Justice et des Affaires sociales.

C'est ainsi par exemple que le Livre Blanc du ministère de la Justice recommande la construction ade petits centres spécialisés sécuritaires» pour la «clientèle exceptionnelle» qu'actuellement, comme on l'admet, «les juges de la Cour du bien-être social refèrent à la cour pour adultes de façon presque automatique». Il s'agit, en somme, de compléter le système déficient de placement par des petites institutions qui vont relever du ministère de la Justice et n'auront aucune autonomie en ce qui a trait aux normes d'admission. On va donc créer, en pratique, des petites prisons pour mineurs particulièrement récalcitrants.

Qu'est-ce que cela signifie de façon plus précise?

On va disposer des petites "prisons" pour mineurs où à cause de leur taille, et des dispositifs sécuritaires les coûts de la construction vont être exorbitants. Comme par ailleurs les ministres changent, mais le ministère des Affaires sociales ne semble pas pour autant particulièrement soucieux d'assumer des responsabilités à 
l'égard du traitement des mineurs délinquants, il est à prévoir que les institutions relevant du ministère de la Justice vont devenir aussitôt des «dépotoirs" privilégiés. Rappelons à cet égard que le Centre Berthelet, devait à l'origine, être utilisé uniquement pour des délinquants difficiles, "à l'exclusion des malades mentaux et des infirmes», pour des périodes «ne dépassant pas trois moisn. En fonction de cette "mission", l'institution a été dotée de barreaux, dignes de prisons pour criminels adultes. Par la suite, cependant, faute de place ailleurs, on y a reçu non seulement des jeunes délinquants, mais aussi des mineurs placés en vertu de la loi de protection de l'enfance malheureuse... et cela pour des périodes très variables. Le manque chronique de place rendait le transfert impossible et plusieurs délinquants juvéniles qui devaient être traités ensuite dans une école de protection, finissaient par être renvoyés chez eux. Le Centre St-Vallier, par contre, prévu comme centre d'accueil pour de courtes périodes d'observation, deuxième institution pour mineurs, obligée de recevoir tous les cas emmenés par la police, a déjà gardé un enfant pendant deux ans. On prétend qu'il a été oublié par les juges, les agents de probation et probablement par le Bon Dieu lui-même...

Est-il vraiment indispensable de recommencer les mêmes expériences, au lieu de créer enfin un système intégré susceptible de répondre aux besoins réels des jeunes et dépendant d'un seul organisme, tel que la Commission de la protection de la jeunesse, par exemple, pour éviter les éternels conflits interministériels, interprofessionnels, interinstitutionnels?

Il ne s'agit plus là des droits des mineurs, mais de la plus élémentaire nécessité d'organiser un système intégré et suffisamment efficace pour éviter aux enfants que la collectivité doit protéger en principe, les humiliations et les traumatismes qu'elle leur impose en fait : ou encore des... suicides ${ }^{2}$.

Pour conclure cet examen schématique et fort incomplet en réalité de la façon suivant laquelle notre collectivité se protège contre les agissements marginaux des mineurs, sans se soucier de la mise en pratique réelle des intentions théoriques contenues dans les législations, il ne nous reste qu'à citer une dernière phrase du Livre Blanc du ministère de la Justice :

2. C'est ainsi qu'à Berthelet, en 1973, deux jeunes que la Société se devait de protéger se sont enlevé la vie. Le rapport de la Commission nommée par le ministère du Bien-Être social, pour faire l'enquête sur ce drame n’a jamais été rendu public. 
L'expérience démontre qu'une confrontation prématurée des jeunes avec le monde criminel adulte occasionne presque automatiquement une récidive et une escalade de la violence. Une intervention positive à l'adolescence pourrait en faire des actifs pour la société.

Selon les statistiques officielles, les prisons du Québec ont reçu en 1974, 1381 mineurs, garçons et filles et il n'est pas du tout certain que la création des "petits centres sécuritaires" va être "une intervention positive».

De toute évidence le ministère de la Justice du Québec a essayé d'améliorer le système de traitement des mineurs, mais le ministère des Affaires sociales, pour sa part, semble être bien plus motivé par le souci d'élargir son champ d'action que par celui de satisfaire les besoins réels des enfants et des familles. Au-delà des textes législatifs, il y a en somme l'évidence de l'insuffisance des politiques du ministère des Affaires sociales du Québec et la nécessité absolue de faire intervenir les organismes communautaires et bénévoles, ne serait-ce que pour faire connaître à l'opinion publique les réalités vécues, chaque jour, par les enfants de malheur...

\section{BIBLIOGRAPHIE}

CONSEIL NATIONAL DU BIEN-ETRE SOCIAL (1975) : les Enfants pauves, Rapport du Conseil national du bien-être social sur les enfants pauvres au Canada, 56 pages.

FONDS DES NATIONS UNIES POUR L'ENFANCE (1971): les Carnets de l'enfance/Assignment Children, Neuilly, 134 pages.

QUÉBEC, MINISTERE DE LA JUSTICE (1974) : la Justice contemporaine, Québec, Éditeur Officiel du Québec, 359 pages.

QUÉBEC, MINISTERE DE LA JUSTICE (1975) : Rapport annuel 1974, Direction générale de la probation et des établissements de détention, 213 pages.

RYAN, T.J. (1972) : Poverty and the Child, Toronto, McGraw-Hill Ryerson, 254 pages.

STATISTIQUE CANADA (1973): Annuaire du Canada, Ottawa, Information Canada, 1041 pages.

SOLLICITEUR GENERAL DU CANADA (1975) : Rapport du Comité du ministère du Solliciteur général du Canada surles propositions formulées en remplacement de la loi sur les jeunes délinquants, Ottawa, 104 pages.

MINISTERE DES AFFAIRES SOCIALES, Avant-projet de loi sur la protection de la jeunesse, Québec, 27 juin 1975, déposé devant l'Assemblèe nationale, 83 pages. 